\title{
La SMSR, une jeune dame de 141 ans
}

SOCIÉTÉ MÉDICALE DE LA SUISSE ROMANDE

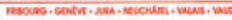

Dr Charles A. Favrod-Coune

Président de la SMSR
Depuis 1867, la Société Médicale de la Suisse Romande rassemble en son sein les sociétés cantonales de médecine francophones de Suisse. Elle est le trait d'union entre cantons romands, mais aussi entre médecins de diverses spécialités, statuts professionnels ou académiques et entre les générations. Elle est le partenaire des instances politiques supra-cantonales en Suisse romande.

\section{Un peu d'histoire}

Originellement dédiées uniquement aux échanges scientifiques, les sociétés cantonales de médecine ont rapidement découvert que l'exercice de la médecine dépendait autant de la politique que des développements de la science. La coordination entre les sociétés cantonales devenait une nécessité. Ainsi furent fondées à bref intervalle la SMSR (1867) et le VEDAG (1870), puis l’Ordine dei Medici (1931). En 1921, la SMSR et le VEDAG fondèrent ensemble la FMH pour représenter leurs intérêts auprès des autorités fédérales.

\section{Activités}

La SMSR exerce fondamentalement sa mission d'échange entre les six sociétés cantonales romandes (JU, NE, FR, VS, VD, GE), sans oublier le Cercle de Pierre-Pertuis (Berne francophone). Des réunions fréquentes entre les présidents de ces sociétés sont le lieu d'échange d'informations et d'ajustage des politiques cantonales, chaque société restant entièrement libre de sa politique.

Depuis la création de l'Assemblée des délégués de la FMH, la SMSR y représente également, avec ses 3 délégués, l'ensemble des cantons romands.

\section{Communication scientifique et professionnelle}

La SMSR publie une revue hebdomadaire, la Revue Médicale Suisse RMS. Ce média a une large audience également hors de Suisse. Il possède de longue date une édition Internet hautement évolutive, à la pointe du progrès dans le domaine de l'e-medicine (www.revmed.ch).

Toujours prête à innover, également avec les éditions Médecine et Hygiène, la SMSR vient de lancer une revue grand public Planète Santé. Trimestriel, il est destiné à donner aux patients et
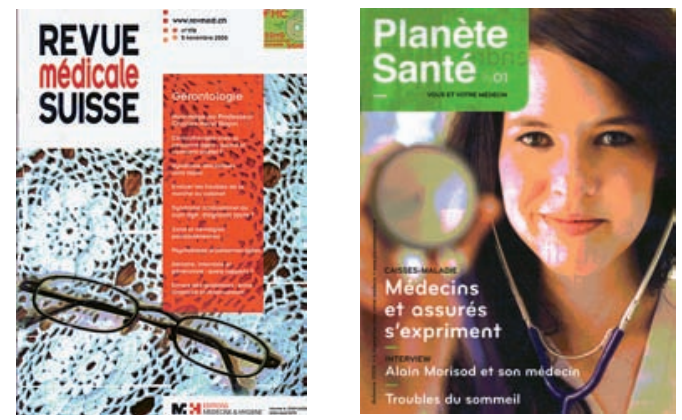

à leurs proches des informations avec l'éclairage des médecins, et non plus seulement des assureurs, des pharma et des politiciens. Il aborde largement les questions de médecine proprement dite et les questions de politique médicale et de santé publique.

\section{Réflexion éthique et politique}

La SMSR organise des conférences de travail thématiques très enrichissantes. Les « 9 pistes pour améliorer l'économicité et la qualité du système de santé suisse» ont été publiées à la suite d'un tel travail. Cette publication reste une référence politique, même si certains chapitres nécessitent aujourd'hui une réactualisation. Ce document est disponible en ligne en français et allemand. (www.smsr.ch/documents)

En 2007 et 2008, des séminaires sur les réseaux de soins (managed care) ont permis à tous de s'informer et d'échanger des idées, ce qui a débouché sur des solutions cantonales originales et diverses. Le dialogue est cherché avec tous partenaires actifs dans la santé, par exemple les sociétés de pharmacie.

\section{Action sociale}

Finalement, signalons le Fonds Louis-Mégevand, un fonds d'aide aux médecins romands dans le besoin et à leurs familles. Son intense activité montre bien sa nécessité, même dans notre «Etat social» moderne.

En résumé, la SMSR est le lieu de convergence, de communication et de synergie des médecins suisses, dans le respect de la pluralité et de la culture romandes. 